\title{
Simultaneous spectra and radio properties of BL Lac's
}

\author{
M. Mingaliev ${ }^{1,2}$, Yu. Sotnikova ${ }^{1 \star}$, T. Mufakharov ${ }^{3}$, E. Nieppola ${ }^{4,5}$, M. Tornikoski ${ }^{4}$, J. Tammi ${ }^{4,6}$, \\ A. Lähteenmäki ${ }^{4,6}$, R. Udovitskiy ${ }^{1}$, and A. Erkenov ${ }^{1}$ \\ 1 Special Astrophysical Observatory of RAS, Nizhnij Arkhyz, 369167 Russia \\ e-mail: marat@sao.ru \\ 2 Kazan Federal University, 18 Kremlyovskaya St., Kazan, 420008, Russia \\ 3 Shanghai Astronomical Observatory, Chinese Academy of Sciences, Shanghai 200030, China \\ 4 Aalto University Metsähovi Radio Observatory, Metsähovintie 114, 02540 Kylmälä, Finland \\ ${ }^{5}$ Finnish Centre of Astronomy with ESO (FINCA), University of Turku, Väisäläntie 20, FI-21500 Piikkiö, Finland \\ 6 Aalto University Department of Radio Science and Engineering, P.O. Box 13000, FI-00076 Aalto, Finland
}

Received XXXX, accepted XXXX

Published online in Astronomische Nachrichten, DOI 10.1002/asna.201713361

Key words galaxies: BL Lacertae objects: general - galaxies: active - radio continuum: galaxies

\begin{abstract}
We present the results of nine years of the blazar observing programme at the RATAN-600 radio telescope (2005-2014). The data were obtained at six frequency bands $(1.1,2.3,4.8,7.7,11.2,21.7 \mathrm{GHz})$ for 290 blazars, mostly BL Lacs. In addition, we used data at $37 \mathrm{GHz}$ obtained quasi-simultaneously with the Metsahovi radio observatory for some sources. The sample includes blazars of three types: high-synchrotron peaked (HSP), low-synchrotron peaked (LSP), and intermediatesynchrotron peaked (ISP). We present several epochs of flux density measurements, simultaneous radio spectra, spectral indices and properties of their variability. The analysis of the radio properties of different classes of blazars showed that LSP and HSP BL Lac blazars are quite different objects on average. LSPs have higher flux densities, flatter spectra and their variability increases as higher frequencies are considered. On the other hand, HSPs are very faint in radio domain, tend to have steep low frequency spectra, and they are less variable than LSPs at all frequencies. Another result is spectral flattening above $7.7 \mathrm{GHz}$ detected in HSPs, while an average LSP spectrum typically remains flat at both the low and high frequency ranges we considered.
\end{abstract}

Copyright line will be provided by the publisher

\section{Introduction}

Active galactic nuclei (AGN) are unique objects in the Universe, because they are compact and extremely luminous. Their main observational properties can be explained by the presence of the super massive black hole at the centre of the galaxies, surrounded by an accretion disk and by fast-moving clouds. They come in different flavors all of which can be explained in terms of the so-called "unified scheme" (Urry \& Padovani 1995), where the major difference between each of them is their orientation relative to the line of sight of the observer.

Blazars are the radio-loud subclass of AGN, characterized by strong non-thermal radiation across the entire electromagnetic spectrum (Kollgaard 1994; Perlman et al. 1998; Strittmatter et al. 1972). Strong variability at different timescales and wavebands is believed to be a result of relativistic motion of non-thermal plasma along the jet, oriented at small angles to the observers line of sight (Blandford \& Rees 1978; Urry \& Padovani 1995). The average value of viewing angle of blazars is about $5^{\circ}$ according to the estimations made by Lister et al. 2013. The spectral energy distribution (SED) of blazars features two

^sjv@sao.ru broad components: the low-energy part (with the peak in the radio/infrared band) is believed to be formed by synchrotron emission and high-energy hump (with the peak in the gamma-rays) is usually explained in terms of inverse Compton radiation (e.g., Sambruna et al. 1996; Sikora et al. 1994) or hadronic processes (e.g., Mücke \& Protheroe 2001; Petropoulou et al. 2015).

Blazars are commonly distinguished as low-synchrotron peaked (LSP), intermediate-synchrotron peaked (ISP), and high-synchrotron peaked (HSP), depending on the peak frequency of the synchrotron component $\left(v_{\text {peak }}^{S}\right)$ of their SED. In this paper we adopt a blazar classification from the Fermi-LAT 3LAC catalogue (Ackermann et al. 2015), which uses the common convention by Abdo et al. 2010 to classify a blazar as an LSP for $v_{\text {peak }}^{S}<10^{14} \mathrm{~Hz}$, ISP for $10^{14} \mathrm{~Hz}<v_{\text {peak }}^{S}<10^{15} \mathrm{~Hz}$, and HSP for $v_{\text {peak }}^{S}>10^{15} \mathrm{~Hz}$.

Blazars are historically divided in two main classes according to the presence or absence of the lines in their optical spectra: BL Lacertae type objects (BL Lacs) have no lines or very weak ones (with the rest frame equivalent width $\mathrm{EW}<5 \AA$ ), while flat-spectrum radio quasars (FSRQs) exhibit normal quasar-like spectra with strong broad emission lines (Stickel et al. 1991; Urry \& Padovani 1995). 
Currently one of the most extensive list of blazars is presented in the Roma-BZCAT catalogue by Massaro et al. (2009). This catalogue is based on multi-frequency surveys and detailed checkout of the literature. The total number of blazars currently listed in the Roma-BZCAT (Edition 5.0 ) is more than 3500 . But only a relatively small number of objects have been intensively observed at many frequencies simultaneously. The spectral coverage of many of them is poor, both in time and in frequency. Few observatories have long term monitoring programmes for blazars at radio wavelengths, Metsähovi Radio Observatory (Teräsranta et al. 2004), University of Michigan Radio Observatory (UMRAO) (Aller et al. 1999), Owens Valley Radio Observatory (OVRO) (Richards et al. 2011) and INAF-Istituto di Radioastronomia in Medicina and Noto Bach et al. 2007) are among them.

The RATAN-600 radio telescope has been monitoring AGN on regular basis for more than 10 years. In this paper we present observational results for 290 blazars obtained at six frequencies from 1.1 to $21.7 \mathrm{GHz}$ with this telescope. The main objective of our research is to obtain multi-frequency information about radio properties of the blazar subgroups by using a single instrument and simultaneous measurements to exclude possible systematic errors as well as variability. This unique data that we collected allowed us to study the spectral properties for relatively large sample of blazars.

\section{Sample}

The sample consists of blazars - BL Lacs, BL Lac candidates, and blazars of an uncertain type - with flux density more than $400 \mathrm{mJy}$ (added to the sample in 2012) and more than $100 \mathrm{mJy}$ (added in 2014) at $1.4 \mathrm{GHz}$, selected from the Roma-BZCAT catalogue1 (Massaro et al. 2009) maintained by the ASI Science Data Center. The first observations with the RATAN-600 started in early 2005 while the last sources were added to the programme in early 2014 . The sample is heterogeneous and contains sources both faint and extremely bright in the radio domain. The median value of the flux density at $1.4 \mathrm{GHz}$ is $0.3 \mathrm{Jy}$; the minimum value is $0.002 \mathrm{Jy}$ at this frequency (MS 0122.1+0903); the maximum is $22.83 \mathrm{Jy}$ (3C84). The inclusion of some FSRQs in this sample can be explained by ambiguity of blazar classification, as pointed out by Marcha et al. (1996) and Antón \& Browne (2005), and some BL Lacs have been classified as FSRQ or vice versa (Shaw et al. 2012). Originally (in 2005) we observed some FSRQs as the BL Lac sources or BL Lac candidates. But in the blazars classification in the BZCATs later edition they were classified as FSRQs and we have included them as such in order to stick to an uniform classification. Since there are both blazars of uncertain type (27) and BL Lac candidates (12) in the sample, it will not affect the analysis of the three subclasses of blazars HSP, ISP

\footnotetext{
1 http://www.asdc.asi.it/bzcat/
}

and LSP. Thus, the sample mainly consists of BL Lacs, BL Lac candidates, blazars of unknown type, and additionally 14 FSRQs.

All objects of the sample were classified as lowsynchrotron peak (LSP), intermediate-synchrotron peak (ISP), and high-synchrotron peak (HSP) blazars: 197 of them were detected by Fermi-LAT and classified according to the 3LAC. For another 93 objects we have used the ASDC SED buildet to fit the synchrotron part of the SED with the second or third degree polynom and get its maximum. The logarithm of the synchrotron peak frequency was calculated in the rest frame. We did not classify two sources in our sample (PGC 59947 and BZB J1733+4519) because they do not have enough observational data points in their SED to extract the reliable $\log v_{\text {peak }}^{S}$ value. The source list includes 149 LSPs, 62 ISPs, 77 HSPs and 2 blazars with unknown SED type. The list of sources and their characteristics are presented in Table 6 .

Col. 1 - Source name,

Col. 2-3 - R.A. and Dec (J2000.0),

Col. 4 - Number of observing epochs at RATAN,

Col. 5 - Redshift $z$,

Col. 6 - Logarithm of the synchrotron peak frequency $v_{\text {peak }}^{S}$ in $\mathrm{Hz}$,

Col. 7 - SED class from 3LAC, an asterisk was used to denote blazars, for which the values of $\log v_{\text {peak }}^{S}$ were calculated using ASDC SED builder,

Col. 8 - AGN class from BZCAT (Edition 5.0) Massaro et al. (2015).

The redshifts for objects were mostly obtained from the Roma-BZCAT, and for some sources from Simbad 3 . Redshifts are known for 238 sources, which is a relatively large number, considering the difficulty of obtaining this information for BL Lacs. The redshift distribution is presented in Fig. 1. The redshift values range from $z=0.018$ to 2.277 , with the mean equal to 0.45 and median to 0.36 . Redshifts have not been measured for 52 objects; 13 of them are HSP objects, 8 are ISPs and 31 are LSPs.

\section{Observations}

The observations were carried out with the RATAN-600 radio telescope during 2005-2014. Systematic monitoring was carried out during the period 2006-2008 for roughly one third of the sources. Observations were done in the transit (meridian) mode with the north and south sectors of the antenna Korolkov \& Pariiskiil 1979; Parijskij 1993). An angular resolution in this mode of observations depends on a declination a source being observed. The FWHM in right ascension (RA) is given in Table 1, an angular resolution in declination is three to five times worse than in RA. Sources were observed from 3 to 15 times in each epoch to increase

\footnotetext{
2 http://tools.asdc.asi.it/SED/

3 http://simbad.u-strasbg.fr/simbad/
} 


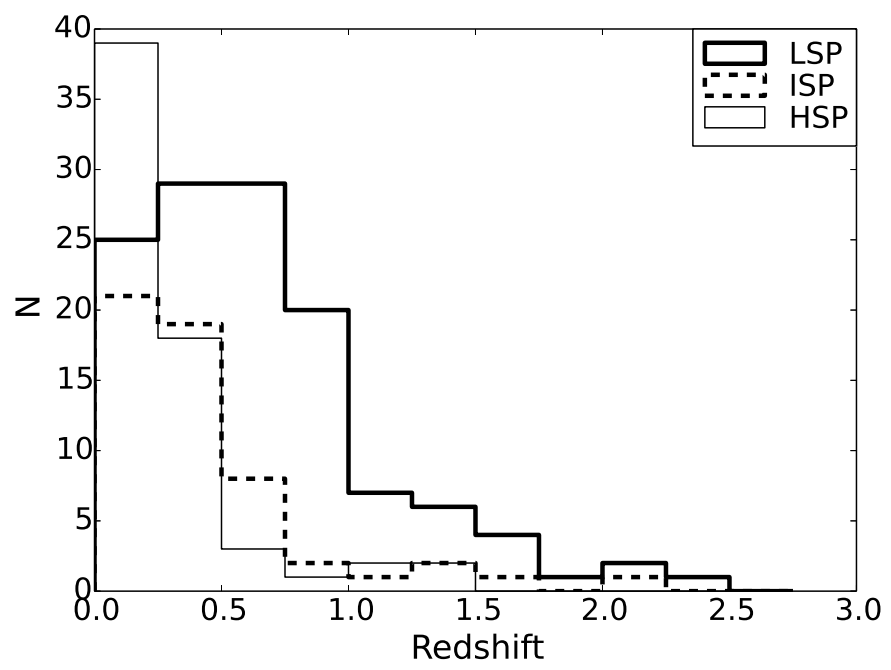

Fig. 1 Redshift distribution for different blazar subclasses of the sample.

the reliability of results and because of the weather or the receiver conditions.

Observation were carried out at six frequencies: 4.8, 7.7, 11.2 , and $21.7 \mathrm{GHz}$ (cryogenically cooled radiometers), and 1.1 and $2.3 \mathrm{GHz}$ (uncooled radiometers). The 4.8 $\mathrm{GHz}$ cooled radiometer is a noise added radiometer (NAR), while other radiometers are designed according to the beamswitching scheme. All the radiometers were designed as the "direct amplification Dicke type" receivers. We use the data acquisition and controling system for all continuum radiometers, as described by Tsybulev (2011).

The experimental data were processed with using the modules of the FADPS (Flexible Astronomical Data Processing System) standard reduction package by Verkhodanov (1997); this is a reduction system for data from the broadband continuum radiometers of the RATAN600 secondary mirror. The processing methods are described in Mingaliev et al. (2007, 2012). The following eight flux density calibrators were used to calculate the calibration coefficients in the scale by Baars et al. (1977): 3C48, 3C138, 3C147, 3C161, 3C286, 3C295, 3C309.1, and NGC 7027. In addition, we used the traditional RATAN600 flux density calibrators at low elevations: J0240-23, $\mathrm{J} 1154-35$, J0521+16, and the source J0410+76 at high elevations. Measurements of calibrators were corrected for angular size and linear polarization (where necessary), following the data from Ott et al. (1994) and Tabara \& Inoue (1980).

The detection limit for RATAN-600 single sector is approximately $8 \mathrm{mJy}$ under good conditions at $4.8 \mathrm{GHz}$ and at an average antenna elevation $\left(\delta \sim 40^{\circ}\right)$. At other frequencies the detection limit is presented in the Table 1 . This value depends on the atmospheric extinction and the effective area on the antenna elevation $H$ (from $10^{\circ}$ up to $90^{\circ}$ above the horizon) at the corresponding frequencies.
Table 1 RATAN-600 continuum radiometers. Where $f_{0}$ - central frequency, $\Delta f_{0}$ - bandwidth, $\Delta F$ - flux density detection limit per beam, and BW - beam width - an angular resolution in RA (an angular resolution in declination is three to five times worse than in RA).

\begin{tabular}{rlcr}
\hline$f_{0}$ & $\Delta f_{0}$ & $\begin{array}{c}\Delta F \\
\mathrm{mJy} / \text { beam }\end{array}$ & $\begin{array}{r}\mathrm{BW} \\
\operatorname{arcsec}\end{array}$ \\
\hline 21.7 & 2.5 & 70 & 11 \\
11.2 & 1.4 & 20 & 16 \\
7.7 & 1.0 & 25 & 22 \\
4.8 & 0.9 & 8 & 36 \\
2.3 & 0.4 & 30 & 80 \\
1.1 & 0.12 & 160 & 170 \\
\hline
\end{tabular}

The $37 \mathrm{GHz}$ observations were made with the $13.7 \mathrm{~m}$ diameter Aalto University Metsähovi radio telescope, which is a radome enclosed Cassegrain type antenna situated in Finland (60 d 13' 04" N, 24 d 23' 35" E). The measurements were made with a $1 \mathrm{GHz}$-band dual beam receiver centered at $36.8 \mathrm{GHz}$. The HEMPT (high electron mobility pseudomorphic transistor) front end operates at room temperature. The observations are Dicke switched ON-ON observations, alternating the source and the sky in each feed horn. A typical integration time to obtain one flux density data point is between 1200 and $1600 \mathrm{~s}$. The detection limit of our telescope at $37 \mathrm{GHz}$ of the order of 0.2 Jy under optimal conditions. Data points with a signal-to-noise ratio $<$ 4 are handled as non-detections. The flux density scale is set by observations of the HII region DR 21. Sources NGC 7027, 3C 274 and 3C 84 are used as secondary calibrators. A detailed description of the data reduction and analysis is given in Teraesranta et al. (1998). The error estimate in the flux density includes the contribution from the measurement rms and the uncertainty of the absolute calibration.

\section{Results}

\subsection{Flux densities}

The flux densities and the instantaneous spectra at several epochs of the sample sources (except the $37 \mathrm{GHz}$ ) are published in the BL Lac database 4 maintained by the Special Astrophysical Observatory. The database is constantly updated with more data which is freely available and is described by Mingaliev et al. (2014).

Almost all sources have complete data at frequencies 4.8 and $7.7 \mathrm{GHz}$. When available, we also added near-simultaneous $37 \mathrm{GHz}$ data from the Aalto University Metsähovi Radio Observatory database. The data were considered near-simultaneous if they were taken within two weeks of the RATAN observation. The occasional absence of data at certain frequencies is a result of data exclusion because of the partial resolution of a source at some frequencies, a source too weak to be measured reliably, a strong

\footnotetext{
4 www.sao.ru/blcat/
} 

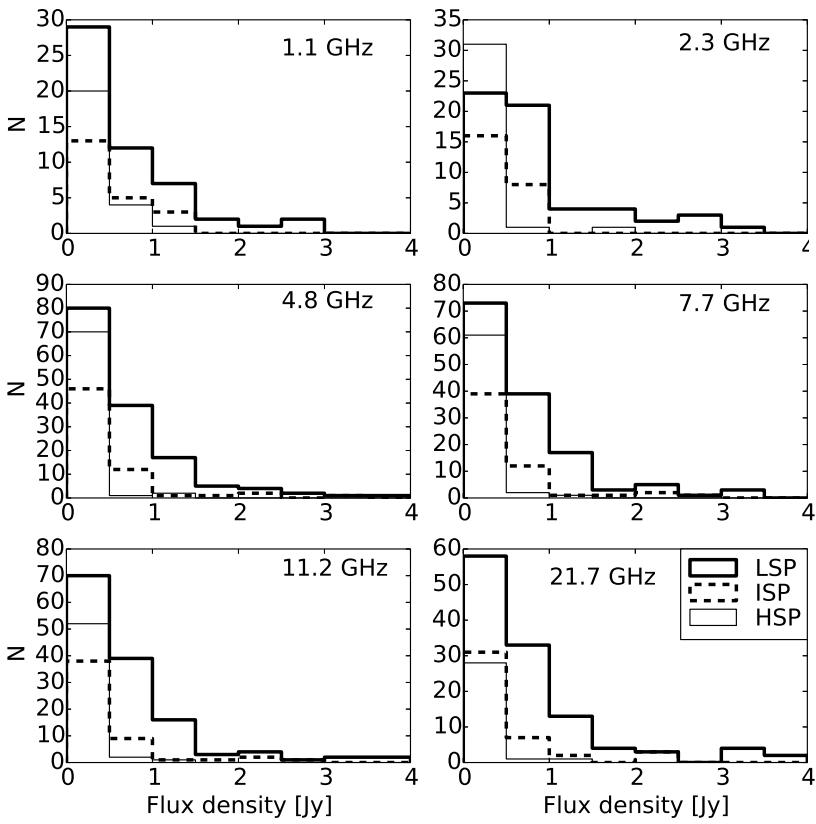

Fig. 2 The average flux density distribution at the RATAN frequencies by SED subclass (some bright sources with the average flux density above $4 \mathrm{Jy}$ are excluded from the plot for the clarity of display).

influence of man-made interferences at 1.1 and $2.3 \mathrm{GHz}$, or due to strong interference from geostationary satellites at 11.2 $\mathrm{GHz}$ (between $-10^{\circ}$ and $0^{\circ}$ degrees of declination). The values of the standard error of fluxes for the most sources are: $5-20 \%$ for $11.2,7.7$, and $4.8 \mathrm{GHz}, 10-35 \%$ for $2.3,1.0$, and $21.7 \mathrm{GHz}$.

The distributions of the average flux densities by source class at all RATAN frequencies are shown in Fig. 2. We used a bin size of 0.5 with the middle value of the bin shown in the graph. The average flux densities have been calculated for $S / N>4$ detections only. The sample sources are typically extremely faint, especially HSPs. Only one HSP (Mrk 501 ) in the sample has an average flux density reaching 1 Jy at most frequencies. This fact underlines the exceptional nature of this sample. Sources this faint are rarely targeted in observational campaigns, and never before have had their radio spectra determined this extensively.

The brightest source in the sample by far is $3 \mathrm{C} 84$ $\left(S_{\text {aver }}=23.18 \mathrm{Jy}\right.$ at $7.7 \mathrm{GHz}$, clearly more than the second brightest source BL Lac with $6.66 \mathrm{Jy}$ ). At $1.1 \mathrm{GHz}$ $1828+487$ is the brightest, but likely only because 3C 84 does not have data at that frequency $(1.1 \mathrm{GHz}$ observations were interrupted in 2012). The flux density of $1828+487$ quickly decreases for frequencies higher than $1.1 \mathrm{GHz}$ due to its steep spectrum. The medians of the average fluxes by frequency and class are listed in Table 2. The average flux density values are not drawn from the same distribution for the different SED classes according to the KruskalWallis test. Pairwise comparisons confirm that LSPs are sig-
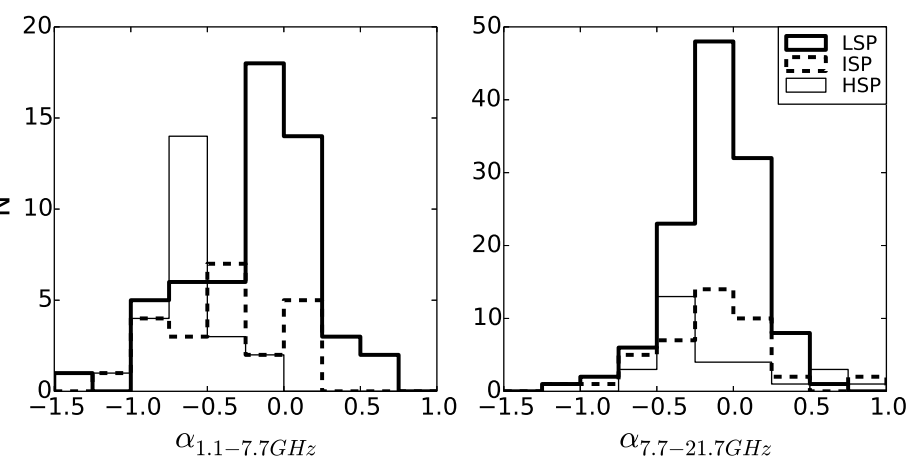

Fig. 3 Distribution of the average low and high frequency spectral indices by SED subclass.

nificantly brighter than HSPs and ISPs at all frequencies $(P<0.001)$.

We included the medians of the flux density values at 37 $\mathrm{GHz}$ in Table 2. These data come from the brightest sources at this frequency, for example, only for $4 \mathrm{HSP}$ objects and 10 ISP. This can obviously bias the interpretation, so the flux at $37 \mathrm{GHz}$ looks high in general.

\subsection{Spectral indices}

We calculated spectral indices for the frequency intervals 1.1-7.7 and 7.7-21.7 GHz to investigate the spectral behavior of blazars at low and high frequencies. We used spectral indices measured in the 1.1-7.7 GHz and in 7.7-21.7 GHz frequency intervals as the base range. We also take the indices in the 2.3-7.7 GHz and in 7.7-11.2 GHz range as low and high frequency spectral indices for some sources that did not have measurements in the base range to increase the significanse of the statistics. The spectral index $\alpha\left(S \propto v^{\alpha}\right)$ was calculated by:

$\alpha=\frac{\log S_{2}-\log S_{1}}{\log v_{2}-\log v_{1}}$,

where $S_{1}$ is the flux density at the frequency $v_{1}$, and $S_{2}$ the flux density at the frequency $v_{2}$. Indices were calculated for $S / N>4$ detections only.

We present results for average and instantaneous low and high frequency spectral indices.

The average spectral indices are listed in Table 3 . Fig. 3 shows the distribution of the average spectral index across the sample. We have found that the spectrum of all three types of blazars we considered flattens at the higher frequencies. The spectral indices are closer to zero and have less scatter in 7.7-21.7 GHz and 11.2-37 GHz intervals in comparison to index measured in $1.1-7.7 \mathrm{GHz}$ frequency range. Some care is required in interpreting this result, because of the data at $37 \mathrm{GHz}$ and relatively high variability indices at 21.7 and $37 \mathrm{GHz}$ (see Table 5 in the next subsection).

Now we will describe results on instantaneous low and high frequency spectral indices for blazars in our sample. 
Table 2 The medians of the flux density values for the SED classes for each frequency, $N$ is the number of sources used to estimate the medians that were calculated for $S / N>4$ detections only (the brightest source $3 C 84$ was excluded from calculation).

\begin{tabular}{lllllllllllllll}
\hline \hline Class & $\begin{array}{l}S_{1.1} \\
{[\mathrm{Jy}]}\end{array}$ & $\mathrm{N}$ & $\begin{array}{l}S_{2.3} \\
{[\mathrm{Jy}]}\end{array}$ & $\mathrm{N}$ & $\begin{array}{l}S_{4.8} \\
{[\mathrm{Jy}]}\end{array}$ & $\mathrm{N}$ & $\begin{array}{l}S_{7.7} \\
{[\mathrm{Jy}]}\end{array}$ & $\mathrm{N}$ & $\begin{array}{l}S_{11.2} \\
{[\mathrm{Jy}]}\end{array}$ & $\mathrm{N}$ & $\begin{array}{l}S_{21.7} \\
{[\mathrm{Jy}]}\end{array}$ & $\mathrm{N}$ & $\begin{array}{l}S_{37} \\
{[\mathrm{Jy}]}\end{array}$ & $\mathrm{N}$ \\
\hline HSP & 0.27 & 25 & 0.09 & 33 & 0.06 & 73 & 0.06 & 64 & 0.06 & 55 & 0.16 & 30 & 0.64 & 4 \\
ISP & 0.53 & 22 & 0.42 & 25 & 0.35 & 63 & 0.31 & 56 & 0.30 & 52 & 0.38 & 43 & 0.63 & 10 \\
LSP & 0.54 & 56 & 0.72 & 61 & 0.68 & 153 & 0.73 & 146 & 0.68 & 141 & 0.71 & 121 & 1.37 & 43 \\
\hline
\end{tabular}

Table 3 Average and median spectral indices by classes for 1.1-7.7, 7.7-21.7 and 7.7-37 GHz frequency intervals. $\mathrm{N}$ - is the number of the instantaneous measurements used for calculations.

\begin{tabular}{llcc}
\hline Class & $\mathrm{N}$ & $\begin{array}{c}\alpha \\
\text { average }\end{array}$ & $\begin{array}{c}\alpha \\
\text { median }\end{array}$ \\
\hline $1.1-7.7 \mathrm{GHz}$ & & & \\
\hline \hline $\mathrm{HSP}$ & 55 & -0.61 & -0.55 \\
$\mathrm{ISP}$ & 65 & -0.28 & -0.26 \\
$\mathrm{LSP}$ & 190 & -0.05 & -0.03 \\
\hline $7.7-21.7 \mathrm{GHz}$ & & & \\
\hline $\mathrm{HSP}$ & 85 & -0.27 & -0.30 \\
ISP & 154 & -0.19 & -0.16 \\
LSP & 668 & -0.12 & -0.10 \\
\hline $7.7-37 \mathrm{GHz}$ & & & \\
\hline HSP & 25 & -0.15 & -0.14 \\
ISP & 29 & 0.03 & -0.01 \\
LSP & 265 & 0.01 & 0.02 \\
\hline
\end{tabular}

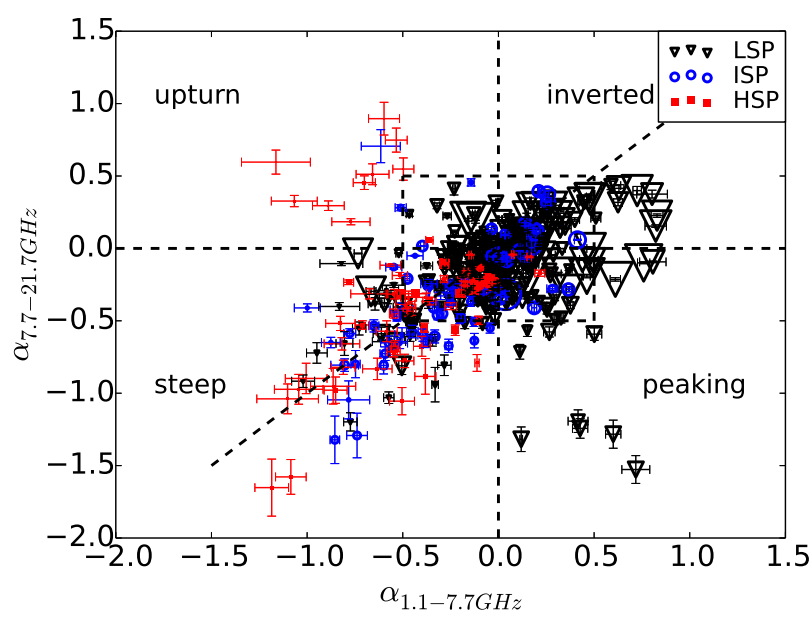

Fig. 4 The "radio colour" plot for instantaneous low and high frequency spectral indices. Black reverse triangles denote LSPs, blue circles - ISPs and red squares for HSPs. The size of the marks is related to the flux density at $7.7 \mathrm{GHz}$.

The instantaneous spectral index term refers to a spectral index obtained at one epoch, thus the number of the instantaneous spectral indices is equal to the number of epochs of observations for each source. We plotted the instantaneous low and high frequency spectral indices in a two-colour diagram (or "radio colour") in Fig. 4. This kind of representation has been previously used by, e.g., Sadler et al. (2006) and Tucci et al. (2008). It is a convenient way to describe the shape of the radio spectrum. The dashed lines divide the diagram into four quadrants signifying a steep, upturning, inverted or peaking spectra as marked in the figure. The dashed inclined line is an one-to-one correspondence line, which is an indicator of the spectral behavior. The position of points relative to this line reflects the character of the spectral slope change (steeper or flatter). The box in the center of the plot denotes an area for the flat spectra (with $\left.\left|\alpha_{\text {radio }}\right|<0.5\right)$, it is usually referred as the "blazar box". Most of the spectra corresponding to LSPs (82\%) and ISPs $(63 \%)$ are flat, while only $41 \%$ of HSPs have a flat spectrum. For $44 \%$ of HSPs and $34 \%$ of the ISPs the low frequency spectrum is, in fact, steep. Table 4 lists the number of objects in each segment of the plot: inside the blazar box (flat spectrum), and in each quadrant outside the blazar box.

For 35 out of 63 HSPs the spectrum turns flatter at 7.7 $\mathrm{GHz}$, i.e., they are situated above the one-to-one correspondence line in Fig. 4 . Nine HSP sources even have a clear upturn. The behaviour of ISPs and LSPs is different: for more than a half of LSPs (183/250) and ISPs (48/70) the spectrum turns steeper at $7.7 \mathrm{GHz}$. HSPs show more often spectral flattening beyond $7.7 \mathrm{GHz}$ (or even inverted), while the LSP spectra get steeper at that point. LSPs and HSPs clearly occupy different regions of the two-colour plot, with LSPs confined to the right edge and reaching high values of spectral indices $\left(\alpha_{\text {radio }}>-0.5\right)$, while HSPs generally fall into "steep" and "upturn" quadrant.

Kruskal-Wallis pairwise comparisons confirm that the distributions of the distances from the one-to-one line are not significantly different either for HSPs and ISPs or ISPs and LSPs, but instead are significantly different for HSPs and LSPs $(P=0.05)$.

The Pearson product-moment correlation coefficient between the instantaneous low and high frequency spectral indices is relatively high and significant only for ISPs $(r=$ 0.60). The Spearman rank correlation coefficient values are: 0.28 for HSPs, 0.63 for ISPs and 0.37 for LSPs; and significant (at the 0.05 level) only for ISPs and LSPs. The correlation means that the typical radio spectrum of ISPs and LSPS can be better approximated with a single power-law, while such a model does not describe HSPs spectra accurately.

From both Fig. 3 and Fig. 4 it is clear that the three SED classes have different distributions of the low and high frequency spectral indices, that fact is confirmed by the 
Table 4 Blazars distribution in the "radio colour" plot in Fig. (4by type of the radio spectrum: flat, inverted, peaking, steep or upturn. "Flat" radio spectrum refers to objects inside the blazar box, with $\left|\alpha_{\text {radio }}\right|<0.5$. The number of measured instantaneous spectral indices for each blazar subclass is presented, the fraction of measurements belonging to the specific spectrum type of is given in brackets.

\begin{tabular}{lccc}
\hline \hline Spectral shape & \multicolumn{3}{c}{ Number of indices } \\
& LSP & ISP & HSP \\
\hline flat & $206(82 \%)$ & $44(63 \%)$ & $26(41 \%)$ \\
inverted & $7(3 \%)$ & $0(0 \%)$ & $0(0 \%)$ \\
peaking & $12(5 \%)$ & $0(0 \%)$ & $0(0 \%)$ \\
steep & $25(10 \%)$ & $24(34 \%)$ & $28(44 \%)$ \\
upturn & $0(0 \%)$ & $2(3 \%)$ & $9(14 \%)$ \\
\hline
\end{tabular}

Kruskall-Wallis test. The difference is also evident in the average spectral indices in Table 3 .

\subsection{Variability}

In order to characterize the variability properties of the sources at the various frequencies we have computed the variability and modulation indices. The first one takes into account measurement uncertainties, while the modulation index is less sensitive to outliers. The variability index was calculated as (Aller et al. 1992):

$V_{S}=\frac{\left(S_{\max }-\sigma_{S_{\max }}\right)-\left(S_{\min }+\sigma_{S_{\text {min }}}\right)}{\left(S_{\max }-\sigma_{S_{\max }}\right)+\left(S_{\min }+\sigma_{S_{\text {min }}}\right)}$

where $S_{\max }$ and $S_{\min }$ are the maximum and minimum value of the flux density, respectively, at all epochs of observations; $\sigma_{S_{\max }}$ and $\sigma_{S_{\min }}$ are their errors. This prevents one from overestimating $V_{S}$ when there are observations with large uncertainties in the dataset. The negative value of $V_{S}$ corresponds to the case where the flux error is greater than the observed scatter in the data.

The modulation index, defined as the standard deviation of the flux density divided by the mean flux density, was calculated as in Kraus et al. 2003:

$M=\frac{\sqrt{\frac{1}{N} \sum_{i=1}^{N}\left(S_{i}-\frac{1}{N} \sum_{i=1}^{N} S_{i}\right)^{2}}}{\frac{1}{N} \sum_{i=1}^{N} S_{i}}$

The medians of the variability and modulation indices for the SED classes by frequency are listed in Table 5. Almost $50 \%$ of HSP objects, $48 \%$ of ISPs, and $56 \%$ of LSPs show variability $M \geq 0.2$. At high frequencies (7.7, 11.2 and $21.7 \mathrm{GHz}$ ) only a small number of each type of blazar show variability above $0.7-0.8$. The most variable object at all frequencies except $1.1 \mathrm{GHz}$ is $\mathrm{AO} 0235+164(27$ epochs at high frequencies). It has a bright peaking spectrum at two epochs due to a passing flare in addition to the generally flat spectrum. A large number of observations increases the probability of finding it in active states, such as
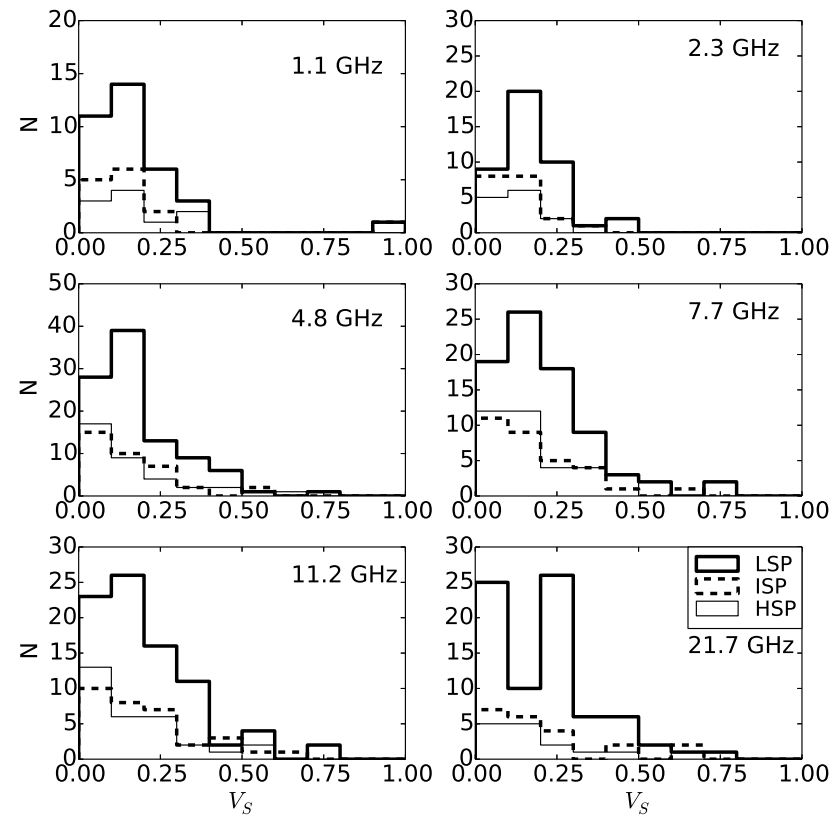

Fig.5 The variability index distributions at the RATAN frequencies by SED subclass.
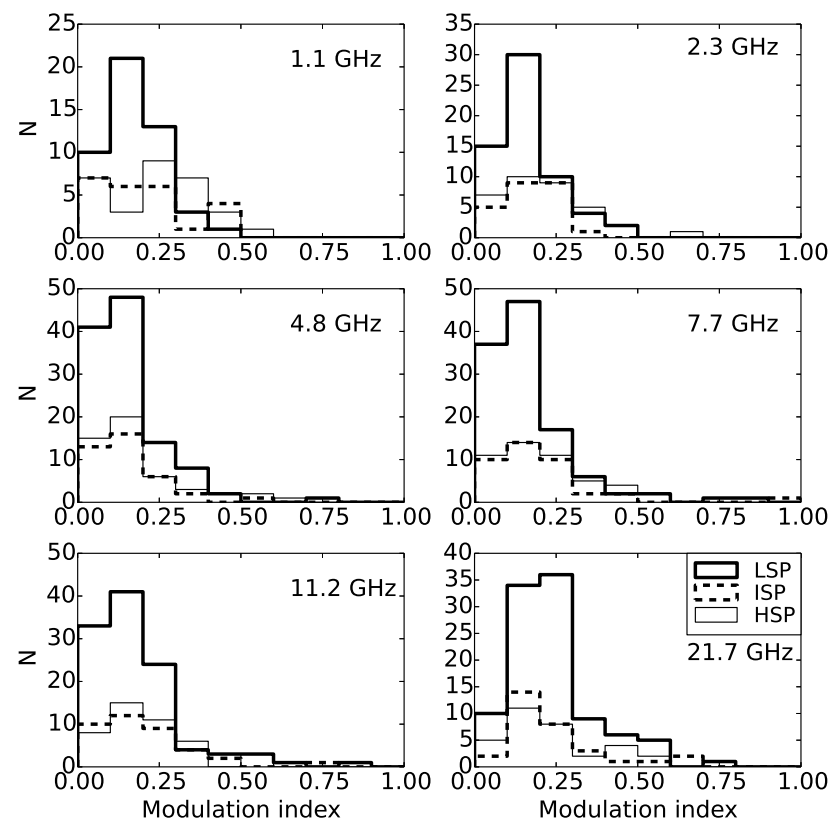

Fig. 6 The modulation index distributions at the RATAN frequencies by SED subclass.

the two peaking spectra contributing to the high variability index. However, at $1.1 \mathrm{GHz}$ the source has little variability. The most variable source at $1.1 \mathrm{GHz}$ is $2 \mathrm{E} 0323+0214$ with $M_{1.1}=0.54$.

The HSPs and ISPs have lower values of the modulation index at six frequencies than LSPs. On the whole, blazars of all three types are less variable at $4.8 \mathrm{GHz}$, according to the median values of both the modulation and the variability indices. The modulation indices are not drawn from the same 
Table 5 The medians of the variability $V_{S}$ and modulation indices $M$ for three blazar subclasses. $\mathrm{N}$ is the number of epochs used to estimate the medians.

\begin{tabular}{|c|c|c|c|c|c|c|c|c|c|c|c|c|c|c|}
\hline \multirow[b]{2}{*}{ Class } & \multicolumn{2}{|c|}{$1.1 \mathrm{GHz}$} & \multicolumn{2}{|c|}{$2.3 \mathrm{GHz}$} & \multicolumn{2}{|c|}{$4.8 \mathrm{GHz}$} & \multicolumn{2}{|c|}{$\overline{7.7 \mathrm{GHz}}$} & \multicolumn{2}{|c|}{$11.2 \mathrm{GHz}$} & \multicolumn{2}{|c|}{$21.7 \mathrm{GHz}$} & \multicolumn{2}{|c|}{$37 \mathrm{GHz}$} \\
\hline & $\mathrm{N}$ & $M$ & $\mathrm{~N}$ & $M$ & $\mathrm{~N}$ & $M$ & $\mathrm{~N}$ & $M$ & $\mathrm{~N}$ & $M$ & $\mathrm{~N}$ & $M$ & $\mathrm{~N}$ & $M$ \\
\hline HSP & 30 & 0.15 & 34 & 0.14 & 49 & 0.11 & 47 & 0.12 & 43 & 0.13 & 32 & 0.19 & 3 & 0.20 \\
\hline ISP & 24 & 0.16 & 24 & 0.14 & 39 & 0.11 & 39 & 0.12 & 38 & 0.12 & 31 & 0.18 & 5 & 0.27 \\
\hline LSP & 48 & 0.17 & 61 & 0.14 & 114 & 0.12 & 113 & 0.13 & 110 & 0.14 & 101 & 0.21 & 30 & 0.21 \\
\hline & $\overline{\mathrm{N}}$ & $\overline{\overline{V_{S}}}$ & $\overline{\bar{N}}$ & $\overline{\overline{V_{S}}}$ & $\overline{\mathrm{N}}$ & $\overline{\bar{V}}$ & $\overline{\mathrm{N}}$ & $\overline{\overline{V_{S}}}$ & $\overline{\mathrm{N}}$ & $\overline{\overline{V_{S}}}$ & $\overline{\mathrm{N}}$ & $\overline{\overline{V_{S}}}$ & $\overline{\bar{N}}$ & $\overline{\overline{V_{S}}}$ \\
\hline HSP & 30 & 0.15 & 34 & 0.10 & 49 & 0.10 & 47 & 0.14 & 43 & 0.11 & 32 & 0.13 & 3 & 0.10 \\
\hline ISP & 24 & 0.15 & 24 & 0.10 & 39 & 0.11 & 39 & 0.13 & 38 & 0.17 & 31 & 0.17 & 5 & 0.19 \\
\hline LSP & 48 & 0.17 & 61 & 0.17 & 114 & 0.14 & 113 & 0.17 & 110 & 0.17 & 101 & 0.20 & 30 & 0.29 \\
\hline
\end{tabular}

distribution only at low frequencies $(1.1$ and $2.3 \mathrm{GHz})$ for the LSP and HSP classes according to the Kruskal-Wallis test.

\section{Discussion}

We find interesting differences between the blazar classes in the radio colour plot (Fig. 4). Almost half of HSPs (28 out of 63) in the sample are found to be steep spectrum sources. Only the minority of LSPs and ISPs (25/250 and 24/70, respectively) fall into "steep" quadrant, the most of them have a flat radio spectrum. The latter is not surprising, because a flat radio spectrum was a standard selection criterion of the early radio-selected BL Lac samples. Most of those sources are today classified as LSPs.

The results obtained in this study indicate that the steep and flattening spectra of HSPs is real. One possibility is that there is an extended component in HSP BL Lacs producing optically thin synchrotron radiation, while the flat radio spectrum of LSP BL Lacs can be explained by the compact nuclear core emission. Chhetri et al. (2012) investigated the dependence between the core/extended morphology and radio spectral indices using $20 \mathrm{GHz}$ data from the Australia Telescope Compact Array for the AT20G sample. They conclude that $\alpha=-0.46$ is an effective division point between the compact and extended sources. They also note that a considerable fraction of the extended sources (i.e., $\alpha<-0.46$ ) show spectral flattening in the higher frequencies. These sources are presumed to be composed of a compact core with an extended component, such as a steepspectrum quiet jet, lobe or a hot spot. At high radio frequencies this steep spectrum component weakens while the compact, flat spectrum component strengthens, and we observe this as spectral flattening. This situation could happen if the jet is not aligned with the observers line of sight, but the viewing angle is still not very big (close to $30^{\circ}$ in case of BL Lacs (Ghisellini et al. (1993); Jackson \& Wall (1999)). That explains the flattening of HSPs in our sample.

The largest variability in synchrotron radiation is observed after the peak. Assuming that the flat radio spectrum is a result of superimposed individual synchrotron components, the variability should, on average, increase toward higher frequencies. That is observed in all three types of blazars in our sample at 4.8-21.7 GHz (for LSPs even until
$37 \mathrm{GHz}$ ) (see Table 5). Secondly, according to our data, both variability and modulation indices for HSPs at all seven frequencies (except the modulation index at $2.3 \mathrm{GHz}$ ) are less than for LSPs. That result is also expected, because sources with flat spectrum are more variable than sources with steep spectrum, this result is also in good agreement with Bolton et al. (2006); Sadler et al. (2006); Tucci et al. (2008).

\section{Summary}

We have presented the results of an observational campaign on BL Lac blazars carried out between 2005 and 2014 with the RATAN-600 radio telescope. The flux densities at frequencies $1.1,2.3,4.8,7.7,11.2$ and $21.7 \mathrm{GHz}$ were measured at several epochs simultaneously. This data set gives us the opportunity to investigate the differences in the radio spectra of all BL Lac types, including the radio-faint HSPs that are rarely targeted by radio studies. We have made two main conclusions:

1. LSP and HSP BL Lac blazars are quite different objects on average. LSPs have higher flux densities (the median of the flux density value is $S_{7.7}=0.73 \mathrm{Jy}$ ), flatter spectra and their variability increases towards higher frequencies. HSPs are very faint in radio domain (the median is $S_{7.7}=0.06 \mathrm{Jy}$ ), tend to have steep low frequency spectra, and they are less variable than LSPs at all frequencies.

2. LSP blazars have flat radio spectra at both low and high frequency ranges that we considered, while the steep low frequency spectra of HSPs (and ISPs) turns flatter above $7.7 \mathrm{GHz}$. But despite the considerable decrease in the spectral index ("flattening") at higher frequencies in HSPs, we note that the spectrum of LSPs is still flatter than HSPs.

Acknowledgements. The RATAN-600 observations were carried out with the financial support of the Ministry of Education and Science of the Russian Federation (14.518.11.7054) and Russian Foundation for Basic Research (12-02-31649). The authors (MGM, TVM) acknowledge support through the Russian Government Program of Competitive Growth of Kazan Federal University. 


\section{References}

Abdo, A. A., Ackermann, M., Agudo, I., et al. 2010, ApJ, 716, 30

Ackermann, M., Ajello, M., Atwood, W. B., et al. 2015, ApJ, 810, 14

Aller, M. F., Aller, H. D., \& Hughes, P. A. 1992, ApJ, 399, 16

Aller, M. F., Aller, H. D., Hughes, P. A., \& Latimer, G. E. 1999, ApJ, 512, 601

Antón, S. \& Browne, I. W. A. 2005, MNRAS, 356, 225

Baars, J. W. M., Genzel, R., Pauliny-Toth, I. I. K., \& Witzel, A. 1977, A\&A, 61, 99

Bach, U., Raiteri, C. M., Villata, M., et al. 2007, A\&A, 464, 175

Blandford, R. D. \& Rees, M. J., eds. 1978, Some comments on radiation mechanisms in Lacertids

Bolton, R. C., Chandler, C. J., Cotter, G., et al. 2006, MNRAS, 370,1556

Chhetri, R., Ekers, R. D., Mahony, E. K., et al. 2012, MNRAS, 422, 2274

Ghisellini, G., Padovani, P., Celotti, A., \& Maraschi, L. 1993, ApJ, 407, 65

Jackson, C. A. \& Wall, J. V. 1999, MNRAS, 304, 160

Kollgaard, R. I. 1994, Vistas in Astronomy, 38, 29

Korolkov, D. V. \& Pariiskii, I. N. 1979, S\&T, 57, 324

Kraus, A., Krichbaum, T. P., Wegner, R., et al. 2003, A\&A, 401, 161

Lister, M. L., Aller, M. F., Aller, H. D., et al. 2013, AJ, 146, 120

Marcha, M. J. M., Browne, I. W. A., Impey, C. D., \& Smith, P. S. 1996, MNRAS, 281, 425

Massaro, E., Giommi, P., Leto, C., et al. 2009, A\&A, 495, 691

Massaro, E., Maselli, A., Leto, C., et al. 2015, Ap\&SS, 357, 75

Mingaliev, M. G., Sotnikova, Y. V., Bursov, N. N., Kardashev, N. S., \& Larionov, M. G. 2007, AZh, 51, 343

Mingaliev, M. G., Sotnikova, Y. V., Torniainen, I., Tornikoski, M., \& Udovitskiy, R. Y. 2012, A\&A, 544, A25

Mingaliev, M. G., Sotnikova, Y. V., Udovitskiy, R. Y., et al. 2014, A\&A, 572, A59

Mücke, A. \& Protheroe, R. J. 2001, Astroparticle Physics, 15, 121

Nieppola, E., Tornikoski, M., \& Valtaoja, E. 2006, A\&A, 445, 441

Ott, M., Witzel, A., Quirrenbach, A., et al. 1994, A\&A, 284, 331

Parijskij, Y. N. 1993, IEEE Antennas and Propagation Magazine, 35,7

Perlman, E. S., Padovani, P., Giommi, P., et al. 1998, AJ, 115, 1253

Petropoulou, M., Dimitrakoudis, S., Padovani, P., Mastichiadis, A., \& Resconi, E. 2015, MNRAS, 448, 2412

Richards, J. L., Max-Moerbeck, W., Pavlidou, V., et al. 2011, ApJS, 194, 29

Sadler, E. M., Ricci, R., Ekers, R. D., et al. 2006, MNRAS, 371, 898

Sambruna, R. M., Maraschi, L., \& Urry, C. M. 1996, ApJ, 463, 444

Shaw, M. S., Romani, R. W., Cotter, G., et al. 2012, ApJ, 748, 49

Sikora, M., Begelman, M. C., \& Rees, M. J. 1994, ApJ, 421, 153

Stickel, M., Padovani, P., Urry, C. M., Fried, J. W., \& Kuehr, H. 1991, ApJ, 374, 431

Strittmatter, P. A., Serkowski, K., Carswell, R., et al. 1972, ApJ, 175, L7

Tabara, H. \& Inoue, M. 1980, A\&AS, 39, 379

Teraesranta, H., Tornikoski, M., Mujunen, A., et al. 1998, A\&AS, 132,305

Teräsranta, H., Achren, J., Hanski, M., et al. 2004, A\&A, 427, 769

Tsybulev, P. G. 2011, Astrophysical Bulletin, 66, 109

Tucci, M., Rubiño-Martin, J. A., Rebolo, R., et al. 2008, MNRAS,
386,1729

Urry, C. M. \& Padovani, P. 1995, PASP, 107, 803

Verkhodanov, O. V. 1997, Astronomical Data Analysis Software and Systems VI, A.S.P. Conference Series, 125, 46 
Table 6 Blazar sample and some parameters.

\begin{tabular}{|c|c|c|c|c|c|c|c|}
\hline Source & R.A. (J2000) & Dec (J2000) & $\mathrm{N}$ & $\mathrm{Z}$ & $\log v_{\text {peak }}^{S}$ & SED class & AGN class \\
\hline NRAO 5 & 00:06:13.9 & $-06: 23: 35$ & 15 & 0.347 & 13.16 & LSP* & BL Lac \\
\hline MS 0011.7+0837 & $00: 14: 19.7$ & $+08: 54: 01$ & 6 & 0.162 & 12.44 & LSP* & BL.gal.domin \\
\hline PKS 0017+200 & $00: 19: 37.8$ & $+20: 21: 45$ & 3 & 0 & 12.32 & LSP & BL Lac \\
\hline PKS 0019+058 & $00: 22: 32.5$ & $+06: 08: 05$ & 16 & 0 & 13.11 & LSP & BL Lac \\
\hline 2MASX J00323309-2849200 & $00: 32: 33.0$ & $-28: 49: 20$ & 1 & 0.324 & 14.70 & ISP & BL Lac \\
\hline RXS J0325.2+1515 & $00: 35: 14.9$ & $+15: 15: 04$ & 8 & 1.280 & 15.10 & HSP & BL Lac \\
\hline 1ES $0037+405$ & $00: 40: 13.7$ & $+40: 50: 04$ & 1 & 0 & 13.16 & LSP* & bl.un \\
\hline RXS J0045.3+2127 & $00: 45: 19.2$ & $+21: 27: 42$ & 1 & 0 & 16.00 & HSP & BL Lac \\
\hline PKS 0047+023 & $00: 49: 43.3$ & $+02: 37: 04$ & 11 & 0 & 13.63 & LSP & BL Lac \\
\hline PKS 0048-097 & $00: 50: 41.2$ & $-09: 29: 06$ & 14 & 0.200 & 14.61 & ISP & BL Lac \\
\hline NPMIG -09.0033 & $00: 56: 20.0$ & $-09: 36: 29$ & 3 & 0.103 & 15.55 & HSP & BL.gal.domin \\
\hline RXS J0058.2+1723 & $00: 58: 16.7$ & $+17: 23: 13$ & 2 & 0 & 16.28 & HSP* & BL Lac \\
\hline B2 $0103+33$ & 01:06:00.2 & $+34: 02: 03$ & 1 & 0.579 & 13.52 & $\mathrm{LSP} *$ & bl.un \\
\hline Q J0109+181 & 01:09:08.1 & $+18: 16: 07$ & 1 & 0.145 & 15.02 & HSP & BL Lac \\
\hline NPMIG +41.0022 & 01:10:04.7 & $+41: 49: 50$ & 1 & 0.096 & 13.37 & LSP* & BL.gal.domin \\
\hline 1FGL J0110.0+6806 & $01: 10: 12.8$ & $+68: 05: 41$ & 1 & 0.290 & 14.86 & ISP & bl.un \\
\hline S4 0108+38 & $01: 11: 37.3$ & $+39: 06: 28$ & 12 & 0.668 & 12.24 & LSP* & bl.un \\
\hline S2 0109+22 & 01:12:05.8 & $+22: 44: 38$ & 1 & 0.265 & 14.33 & ISP & BL Lac \\
\hline PKS 0118-272 & 01:20:31.6 & $-27: 01: 24$ & 3 & 0.560 & 14.33 & ISP & BL Lac \\
\hline $1 \mathrm{ES} 0120+340$ & 01:23:08.5 & $+34: 20: 47$ & 1 & 0.272 & 17.46 & HSP & BL Lac \\
\hline MS 0122.1+0903 & $01: 24: 44.5$ & $+09: 18: 49$ & 3 & 0.338 & 13.75 & LSP* & BL Lac \\
\hline PKS 0138-097 & $01: 41: 25.8$ & $-09: 28: 43$ & 13 & 0.733 & 13.04 & LSP & BL Lac \\
\hline PKS 0140-059 & $01: 42: 38.8$ & $-05: 44: 01$ & 1 & 0 & 13.56 & LSP* & BL Lac \\
\hline 1ES $0145+138$ & $01: 48: 29.7$ & $+14: 02: 18$ & 8 & 0.125 & 14.27 & ISP & BL.gal.domin \\
\hline $8 \mathrm{C} 0149+710$ & $01: 53: 25.8$ & $+71: 15: 06$ & 2 & 0.022 & 15.69 & HSP & BL.gal.domin \\
\hline 87GB 0156.9+1032 & 01:59:34.4 & $+10: 47: 07$ & 6 & 0.195 & 16.03 & HSP & BL Lac \\
\hline MS 0158.5+0019 & 02:01:06.1 & $+00: 34: 00$ & 4 & 0.298 & 16.82 & HSP* & BL Lac \\
\hline PKS 0202+14 & 02:04:50.4 & $+15: 14: 11$ & 4 & 0.833 & 12.71 & LSP & bl.un \\
\hline S5 $0205+72$ & 02:09:51.7 & $+72: 29: 26$ & 1 & 0.895 & 12.71 & LSP* & bl.un \\
\hline Z $0214+083$ & $02: 17: 17.0$ & $+08: 37: 03$ & 7 & 1.400 & 13.79 & LSP & BL Lac \\
\hline OD 330 & $02: 21: 05.5$ & $+35: 56: 13$ & 2 & 0.944 & 13.16 & LSP & bl.un \\
\hline PKS 0219-164 & 02:22:00.7 & $-16: 15: 16$ & 1 & 0.698 & 14.58 & ISP & FSRQ \\
\hline $3 \mathrm{C} 66 \mathrm{~A}$ & $02: 22: 39.6$ & $+43: 02: 07$ & 5 & 0.444 & 15.09 & HSP & BL Lac \\
\hline $1 \mathrm{ES} 0229+200$ & $02: 32: 48.6$ & $+20: 17: 17$ & 5 & 0.140 & 15.48 & HSP & BL.gal.domin \\
\hline AO $0235+164$ & $02: 38: 38.8$ & $+16: 36: 59$ & 28 & 0.940 & 13.31 & LSP & BL Lac \\
\hline PKS 0245-167 & 02:48:07.7 & $-16: 31: 46$ & 3 & 0 & 12.84 & LSP* & BL Lac \\
\hline RXS J0250.6+1712 & 02:50:38.0 & $+17: 12: 08$ & 7 & 1.100 & 16.41 & HSP & bl.un \\
\hline PKS 0301-243 & 03:03:26.5 & $-24: 07: 13$ & 5 & 0.260 & 15.43 & HSP & BL Lac \\
\hline $4 C 47.08$ & 03:03:35.2 & $+47: 16: 16$ & 5 & 0.475 & 14.00 & ISP & BL Lac \\
\hline PKS 0306+102 & 03:09:03.6 & $+10: 29: 16$ & 15 & 0.863 & 13.14 & LSP & FSRQ \\
\hline RXS J0314.3+0620 & $03: 14: 23.9$ & $+06: 19: 57$ & 5 & 0 & 15.92 & HSP* & BL Lac \\
\hline RXS J0316.1+0904 & 03:16:12.9 & $+09: 04: 43$ & 6 & 0 & 15.77 & ISP & BL Lac \\
\hline $3 C 84$ & 03:19:48.1 & $+41: 30: 42$ & 6 & 0.018 & 13.57 & LSP & bl.un \\
\hline MS $0317.0+1834$ & 03:19:51.8 & $+18: 45: 35$ & 6 & 0.190 & 16.99 & HSP & BL.gal.domin \\
\hline 2E $0323+0214$ & 03:26:13.9 & $+02: 25: 14$ & 7 & 0.147 & 15.93 & HSP & BL Lac \\
\hline PKS 0338-214 & $03: 40: 35.6$ & $-21: 19: 31$ & 5 & 0.223 & 13.49 & LSP & BL Lac \\
\hline PKS 0346-163 & $03: 48: 39.2$ & $-16: 10: 17$ & 8 & 0 & 13.45 & LSP & BL Lac \\
\hline S5 $0346+800$ & 03:54:46.1 & $+80: 09: 28$ & 1 & 0 & 12.60 & LSP & cand. \\
\hline PKS 0357-264 & 03:59:33.6 & $-26: 15: 31$ & 3 & 1.470 & 13.16 & LSP & BL Lac \\
\hline PKS 0406+121 & 04:09:22.1 & $+12: 17: 39$ & 19 & 1.020 & 13.16 & LSP* & BL Lac \\
\hline 2E $0414+0057$ & $04: 16: 52.4$ & $+01: 05: 24$ & 6 & 0.287 & 16.64 & HSP & BL Lac \\
\hline MS 0419.3+1943 & $04: 22: 18.5$ & $+19: 50: 53$ & 2 & 0.512 & 12.62 & LSP* & BL Lac \\
\hline PKS 0420+022 & $04: 22: 52.2$ & $+02: 19: 27$ & 9 & 2.277 & 12.35 & LSP* & FSRQ \\
\hline PKS 0422+004 & $04: 24: 46.8$ & $+00: 36: 07$ & 14 & 0.310 & 14.10 & ISP & BL Lac \\
\hline MCG -01.12.005 & $04: 25: 51.3$ & $-08: 33: 39$ & 4 & 0.039 & 12.80 & $\mathrm{LSP} *$ & BL.gal.domin \\
\hline $3 \mathrm{C} 120$ & $04: 33: 11.0$ & $+05: 21: 15$ & 5 & 0.033 & 14.30 & ISP* & bl.un \\
\hline 2EG J0432+2910 & $04: 33: 37.8$ & $+29: 05: 55$ & 2 & 0.970 & 13.40 & LSP & BL Lac \\
\hline PKS 0439-299 & $04: 41: 19.5$ & $-29: 52: 35$ & 2 & 0 & 13.91 & LSP* & cand. \\
\hline PKS 0446+11 & 04:49:07.6 & $+11: 21: 28$ & 20 & 1.207 & 12.76 & LSP & FSRQ \\
\hline
\end{tabular}


Table 6 Blazar sample and some parameters. Continued.

\begin{tabular}{|c|c|c|c|c|c|c|c|}
\hline Source & R.A. (J2000) & $\operatorname{Dec}(\mathrm{J} 2000)$ & $\mathrm{N}$ & $\mathrm{Z}$ & $\log v_{\text {peak }}^{S}$ & SED class & AGN class \\
\hline PKS 0459+135 & $05: 02: 33.2$ & $+13: 38: 11$ & 8 & 0 & 13.18 & LSP* & BL Lac \\
\hline Q $0458+6530$ & 05:03:05.8 & $+65: 34: 01$ & 1 & 0 & 16.45 & HSP* & cand. \\
\hline RXS J0505.5+0416 & 05:05:34.7 & $+04: 15: 54$ & 6 & 0.027 & 15.96 & HSP & BL Lac \\
\hline 1ES $0502+675$ & 05:07:56.1 & $+67: 37: 24$ & 6 & 0.416 & 18.20 & HSP* & BL Lac \\
\hline S5 $0454+84$ & $05: 08: 42.3$ & $+84: 32: 04$ & 3 & 1.340 & 13.28 & LSP* & BL Lac \\
\hline MG 0509+0541 & 05:09:25.9 & $+05: 41: 35$ & 3 & 0 & 14.22 & ISP & BL Lac \\
\hline 4U 0506-03 & 05:09:39.0 & $-04: 00: 36$ & 14 & 0.304 & 17.13 & HSP* & cand. \\
\hline 1FGL J0515.2+7355 & $05: 16: 31.2$ & $+73: 51: 08$ & 1 & 0.249 & 16.02 & HSP & BL Lac \\
\hline PKS 0524+034 & $05: 27: 32.7$ & $+03: 31: 31$ & 7 & 0 & 12.94 & LSP* & BL Lac \\
\hline 1WGA J0536.4-3342 & 05:36:29.1 & $-33: 43: 02$ & 1 & 0 & 16.05 & $\mathrm{HSP}^{*}$ & BL Lac \\
\hline HB89 0548-322 & $05: 50: 40.6$ & $-32: 16: 17$ & 1 & 0.069 & 17.23 & HSP* & BL.gal.domin \\
\hline B3 0609+413 & $06: 12: 51.1$ & $+41: 22: 37$ & 2 & 0 & 14.49 & ISP & BL Lac \\
\hline MS 0607.9+7108 & $06: 13: 43.3$ & $+71: 07: 26$ & 1 & 0.267 & 15.00 & ISP* & BL Lac \\
\hline $\mathrm{J} 0617+5701$ & $06: 17: 16.9$ & $+57: 01: 16$ & 2 & 0 & 13.45 & LSP & BL Lac \\
\hline 87 GB $06216+4441$ & $06: 25: 18.2$ & $+44: 40: 01$ & 7 & 0 & 14.55 & ISP & BL Lac \\
\hline PKS 0627-199 & $06: 29: 23.7$ & $-19: 59: 19$ & 1 & 0 & 13.23 & LSP & BL Lac \\
\hline 1ES $0647+250$ & $06: 50: 46.5$ & $+25: 03: 00$ & 2 & 0.203 & 16.42 & HSP & BL Lac \\
\hline B3 $0651+428$ & $06: 54: 43.5$ & $+42: 47: 58$ & 1 & 0.126 & 13.01 & LSP* & BL.gal.domin \\
\hline $4 \mathrm{C}+42.22$ & $06: 56: 10.6$ & $+42: 37: 02$ & 5 & 0.059 & 15.39 & HSP & BL.gal.domin \\
\hline 2MASS J06562263-2403194 & $06: 56: 22.6$ & $-24: 03: 19$ & 1 & 0.371 & 15.30 & HSP* & BL Lac \\
\hline $\mathrm{J} 0707+6110$ & 07:07:00.6 & $+61: 10: 11$ & 3 & 0 & 13.48 & LSP* & BL Lac \\
\hline EXO $0706.1+5913$ & $07: 10: 30.1$ & $+59: 08: 21$ & 6 & 0.125 & 16.99 & HSP & BL Lac \\
\hline B3 $0707+476$ & $07: 10: 46.1$ & $+47: 32: 11$ & 2 & 1.292 & 13.24 & ISP & BL Lac \\
\hline GB2 $0716+332$ & 07:19:19.4 & $+33: 07: 09$ & 1 & 0.779 & 14.08 & ISP & FSRQ \\
\hline S5 $0716+714$ & $07: 21: 53.4$ & $+71: 20: 36$ & 6 & 0.300 & 13.99 & LSP & BL Lac \\
\hline PKS 0723-008 & $07: 25: 50.6$ & $-00: 54: 56$ & 4 & 0.128 & 13.41 & LSP & bl.un \\
\hline PKS $0735+17$ & 07:38:07.4 & $+17: 42: 19$ & 15 & 0.424 & 13.98 & LSP & BL Lac \\
\hline $4 \mathrm{C}+54.15$ & 07:53:01.3 & $+53: 52: 59$ & 3 & 0.200 & 13.28 & LSP & BL Lac \\
\hline GB $0751+485$ & $07: 54: 45.6$ & $+48: 23: 50$ & 1 & 0.377 & 13.84 & LSP & BL Lac \\
\hline PKS $0754+100$ & $07: 57: 06.7$ & $+09: 56: 35$ & 24 & 0.266 & 13.49 & LSP & BL Lac \\
\hline PKS 0808+019 & $08: 11: 26.6$ & $+01: 46: 52$ & 16 & 0.930 & 13.23 & LSP & BL Lac \\
\hline 1WGA J0816.0-0736 & $08: 16: 04.3$ & $-07: 35: 59$ & 4 & 0.040 & 14.76 & ISP & BL.gal.domin \\
\hline J0817-0933 & $08: 17: 49.7$ & $-09: 33: 30$ & 2 & 0 & 14.05 & ISP & BL Lac \\
\hline OJ 425 & $08: 18: 16.0$ & $+42: 22: 45$ & 5 & 0.530 & 12.99 & LSP & BL Lac \\
\hline PKS 0818-128 & $08: 20: 57.4$ & $-12: 58: 59$ & 6 & 0.074 & 14.72 & ISP & BL Lac \\
\hline 4C 22.21 & $08: 23: 24.7$ & $+22: 23: 03$ & 4 & 0.951 & 13.27 & LSP* & BL Lac \\
\hline PKS $0823+033$ & $08: 25: 50.3$ & $+03: 09: 24$ & 22 & 0.506 & 13.18 & LSP & BL Lac \\
\hline PKS 0823-223 & $08: 26: 01.5$ & $-22: 30: 27$ & 3 & 0.910 & 14.44 & ISP & BL Lac \\
\hline PKS 0829+046 & $08: 31: 48.9$ & $+04: 29: 39$ & 7 & 0.174 & 13.71 & LSP & BL Lac \\
\hline $1 \mathrm{H} 0827+089$ & $08: 31: 55.1$ & $+08: 47: 43$ & 5 & 0.941 & 13.15 & LSP* & FSRQ \\
\hline OJ 448 & $08: 32: 23.2$ & $+49: 13: 20$ & 2 & 0.548 & 12.90 & LSP & BL Lac \\
\hline TEX $0836+182$ & 08:39:30.7 & $+18: 02: 47$ & 14 & 0.280 & 14.22 & ISP & BL Lac \\
\hline PKS $0837+035$ & $08: 39: 49.2$ & $+03: 19: 53$ & 5 & 1.570 & 12.79 & LSP & BL Lac \\
\hline RXS J0847.2+1133 & $08: 47: 12.9$ & $+11: 33: 52$ & 4 & 0.198 & 15.95 & HSP & BL Lac \\
\hline 2MASS J08475674-0703169 & $08: 47: 56.7$ & $-07: 03: 17$ & 1 & 0 & 13.53 & LSP & BL Lac \\
\hline US1889 & 08:54:09.8 & $+44: 08: 30$ & 3 & 0.382 & 14.16 & ISP & BL Lac \\
\hline OJ 287 & $08: 54: 48.8$ & $+20: 06: 30$ & 11 & 0.306 & 13.73 & LSP & BL Lac \\
\hline NPM1G -09.0307 & 09:08:02.2 & $-09: 59: 37$ & 7 & 0.054 & 12.91 & LSP* & bl.un \\
\hline $3 \mathrm{C} 216.0$ & 09:09:33.4 & $+42: 53: 46$ & 7 & 0.670 & 13.31 & LSP* & bl.un \\
\hline RXS J09130-2103 & 09:13:00.1 & $-21: 03: 20$ & 1 & 0.198 & 16.50 & HSP & BL Lac \\
\hline B2 $0912+29$ & $09: 15: 52.3$ & $+29: 33: 24$ & 3 & 0.101 & 15.59 & HSP & BL Lac \\
\hline B2 $0922+31 \mathrm{~B}$ & $09: 25: 43.6$ & $+31: 27: 10$ & 1 & 0.260 & 13.27 & LSP & BL Lac \\
\hline J09291544+5013360 & 09:29:15.4 & $+50: 13: 35$ & 4 & 0.370 & 14.14 & ISP & BL Lac \\
\hline $1 \mathrm{ES} 0927+500$ & $09: 30: 37.5$ & $+49: 50: 25$ & 5 & 0.188 & 15.29 & HSP & BL Lac \\
\hline B2 $0927+35$ & 09:30:55.2 & $+35: 03: 37$ & 10 & 0 & 12.67 & LSP* & BL Lac \\
\hline B2 $0937+26$ & 09:40:14.7 & $+26: 03: 29$ & 5 & 0.498 & 13.33 & LSP* & BL Lac \\
\hline RXS J09449-1347 & 09:44:59.2 & $-13: 47: 51$ & 1 & 0 & 14.24 & ISP* & cand. \\
\hline 2FGL J0945.9+5751 & $09: 45: 42.2$ & $+57: 57: 47$ & 1 & 0.229 & 14.73 & ISP & BL Lac \\
\hline RXS J09530-0840 & 09:53:02.6 & $-08: 40: 18$ & 1 & 0 & 15.29 & HSP & BL Lac \\
\hline S4 0954+65 & $09: 58: 47.2$ & $+65: 33: 54$ & 6 & 0.367 & 13.49 & LSP & BL Lac \\
\hline
\end{tabular}


Table 6 Blazar sample and some parameters. Continued.

\begin{tabular}{|c|c|c|c|c|c|c|c|}
\hline Source & R.A. (J2000) & Dec (J2000) & $\mathrm{N}$ & $\mathrm{z}$ & $\log v_{\text {peak }}^{S}$ & SED class & AGN class \\
\hline $4 C 22.25$ & $10: 00: 21.9$ & $+22: 33: 18$ & 7 & 0.419 & 14.39 & ISP* & bl.un \\
\hline $\mathrm{J} 1008+0621$ & 10:08:00.8 & $+06: 21: 21$ & 2 & 0.650 & 14.21 & ISP* & BL Lac \\
\hline RXS J1008.1+4705 & 10:08:11.3 & $+47: 05: 20$ & 3 & 0.343 & 14.49 & ISP & BL Lac \\
\hline PKS $1008+013$ & $10: 11: 15.6$ & $+01: 06: 42$ & 1 & 1.275 & 13.01 & LSP* & BL Lac \\
\hline NRAO 350 & $10: 12: 13.3$ & $+06: 30: 57$ & 11 & 0.727 & 14.98 & ISP & BL Lac \\
\hline RXS J1012.7+4229 & $10: 12: 44.3$ & $+42: 29: 57$ & 8 & 0.364 & 16.81 & HSP & BL Lac \\
\hline 2FGL J1019.8+6322 & $10: 19: 50.8$ & $+63: 20: 01$ & 1 & 2.025 & 13.24 & LSP & BL Lac \\
\hline RXS J1022.7-0112 & $10: 22: 43.9$ & $-01: 13: 02$ & 6 & 0 & 16.64 & HSP & BL Lac \\
\hline SDSS J10326+6623 & $10: 32: 39.0$ & $+66: 23: 23$ & 1 & 2.212 & 14.08 & ISP & BL Lac \\
\hline $\mathrm{J} 1036+1233$ & $10: 36: 40.3$ & $+12: 33: 38$ & 1 & 0 & 14.35 & ISP* & BL Lac \\
\hline TEX $1040+244$ & $10: 43: 09.0$ & $+24: 08: 35$ & 15 & 0.560 & 12.77 & LSP & FSRQ \\
\hline S5 $1044+719$ & $10: 48: 27.6$ & $+71: 43: 35$ & 2 & 1.150 & 13.19 & LSP & bl.un \\
\hline GB6 J1054+2210 & $10: 54: 30.6$ & $+22: 10: 54$ & 1 & 1.539 & 14.60 & ISP & BL Lac \\
\hline RX J10578-2753 & $10: 57: 50.7$ & $-27: 54: 11$ & 1 & 0.092 & 15.64 & HSP & BL Lac \\
\hline B3 $1055+433$ & 10:58:02.9 & $+43: 04: 41$ & 1 & 2.204 & 12.68 & $\mathrm{LSP}^{*}$ & BL Lac \\
\hline $4 C 01.28$ & $10: 58: 29.6$ & $+01: 33: 58$ & 8 & 0.890 & 13.18 & LSP & bl.un \\
\hline MRK 421 & $11: 04: 27.2$ & $+38: 12: 32$ & 13 & 0.031 & 17.07 & HSP & BL Lac \\
\hline RXS J1110.6+7133 & $11: 10: 37.5$ & $+71: 33: 56$ & 1 & 0 & 15.55 & HSP & BL Lac \\
\hline FIRST J1117.6+2548 & $11: 17: 40.4$ & $+25: 48: 46$ & 1 & 0.360 & 15.60 & HSP & BL Lac \\
\hline EXO 1118 & $11: 20: 48.0$ & $+42: 12: 12$ & 1 & 0.124 & 17.18 & HSP & BL Lac \\
\hline CGRaBS J1121-0711 & $11: 21: 42.1$ & $-07: 11: 06$ & 1 & 0 & 12.44 & $\mathrm{LSP}^{*}$ & BL Lac \\
\hline $\mathrm{J} 112402.70+23$ & $11: 24: 02.7$ & $+23: 36: 45$ & 5 & 1.549 & 13.12 & LSP & FSRQ \\
\hline $\mathrm{J} 1132+0034$ & $11: 32: 45.6$ & $+00: 34: 27$ & 4 & 1.223 & 14.07 & ISP & BL Lac \\
\hline MS $1133.7+1618$ & $11: 36: 17.6$ & $+16: 01: 53$ & 3 & 0.574 & 12.16 & LSP* & BL Lac \\
\hline MRK 180 & $11: 36: 26.4$ & $+70: 09: 27$ & 1 & 0.045 & 15.77 & HSP & BL Lac \\
\hline A $1137+1544$ & $11: 40: 23.4$ & $+15: 28: 09$ & 1 & 0.244 & 15.99 & HSP & BL Lac \\
\hline GB6 B1144+3517 & $11: 47: 22.1$ & $+35: 01: 07$ & 2 & 0.063 & 14.60 & ISP* & BL.gal.domin \\
\hline $\mathrm{J} 1148+1840$ & $11: 48: 37.7$ & $+18: 40: 09$ & 5 & 0.405 & 13.10 & LSP* & BL Lac \\
\hline EXO 1149.9+2455 & $11: 49: 30.3$ & $+24: 39: 27$ & 3 & 0.402 & 14.61 & ISP* & BL Lac \\
\hline B2 $1147+24$ & $11: 50: 19.2$ & $+24: 17: 54$ & 15 & 0.200 & 14.02 & ISP & BL Lac \\
\hline RXS J1151.4+5859 & $11: 51: 24.6$ & $+58: 59: 17$ & 1 & 0.118 & 14.72 & ISP & BL Lac \\
\hline SBS $1200+608$ & $12: 03: 03.5$ & $+60: 31: 19$ & 1 & 0.065 & 14.93 & ISP & BL Lac \\
\hline $\mathrm{J} 1206+0529$ & $12: 06: 58.0$ & $+05: 29: 52$ & 4 & 0.791 & 13.85 & LSP* & BL Lac \\
\hline CGRaBS J1209-2032 & $12: 09: 14.6$ & $-20: 32: 39$ & 1 & 0.404 & 12.20 & LSP* & BL Lac \\
\hline B3 $1206+416$ & $12: 09: 22.7$ & $+41: 19: 41$ & 1 & 0.377 & 13.85 & LSP & BL Lac \\
\hline 1ES $1212+078$ & $12: 15: 10.9$ & $+07: 32: 04$ & 5 & 0.136 & 13.27 & $\mathrm{LSP}^{*}$ & BL.gal.domin \\
\hline GB6 B1215+3023 & $12: 17: 52.0$ & $+30: 07: 00$ & 3 & 0.130 & 15.26 & HSP & BL Lac \\
\hline PKS 1215-002 & $12: 17: 58.7$ & $-00: 29: 46$ & 1 & 0.419 & 13.51 & LSP & BL Lac \\
\hline GB2 $1217+348$ & $12: 20: 08.2$ & $+34: 31: 21$ & 1 & 0.643 & 13.95 & LSP & BL Lac \\
\hline PG $1218+304$ & $12: 21: 21.9$ & $+30: 10: 37$ & 5 & 0.182 & 16.66 & HSP & BL Lac \\
\hline 1WGA J1221.5+2813 & $12: 21: 31.6$ & $+28: 13: 58$ & 6 & 0.102 & 14.44 & ISP & BL Lac \\
\hline S5 $1221+80$ & $12: 23: 40.4$ & $+80: 40: 04$ & 5 & 0 & 13.22 & LSP & BL Lac \\
\hline RXS J12302+2517 & $12: 30: 14.0$ & $+25: 18: 06$ & 6 & 0.135 & 14.91 & ISP & BL Lac \\
\hline $2 \mathrm{E} 1258+1437$ & $12: 31: 23.9$ & $+14: 21: 25$ & 2 & 0.260 & 15.03 & HSP & BL Lac \\
\hline BZB J1235+1700 & $12: 35: 28.8$ & $+17: 00: 36$ & 1 & 0.381 & 14.01 & ISP* & BL.gal.domin \\
\hline FIRST J1236.3+3900 & $12: 36: 23.0$ & $+39: 00: 01$ & 1 & 0.390 & 14.78 & ISP & BL Lac \\
\hline RX J12370+3020 & $12: 37: 05.5$ & $+30: 20: 05$ & 1 & 0.700 & 12.20 & LSP* & BL Lac \\
\hline RXS J12416+3440 & $12: 41: 41.4$ & $+34: 40: 31$ & 7 & 0 & 12.27 & LSP* & BL Lac \\
\hline 1ES $1239+069$ & $12: 41: 48.3$ & $+06: 36: 01$ & 3 & 0.150 & 15.06 & HSP & BL Lac \\
\hline RX J12418-1455 & $12: 41: 49.3$ & $-14: 55: 58$ & 1 & 0 & 15.88 & HSP & BL Lac \\
\hline Ton 116 & $12: 43: 12.7$ & $+36: 27: 43$ & 3 & 1.065 & 16.15 & HSP & BL Lac \\
\hline S5 $1250+53$ & $12: 53: 11.9$ & $+53: 01: 11$ & 5 & 1.084 & 13.94 & LSP & BL Lac \\
\hline PKS 1256-229 & $12: 59: 08.4$ & $-23: 10: 38$ & 1 & 0.481 & 13.62 & LSP & bl.un \\
\hline
\end{tabular}


Table 6 Blazar sample and some parameters. Continued.

\begin{tabular}{|c|c|c|c|c|c|c|c|}
\hline Source & R.A. (J2000) & Dec (J2000) & $\mathrm{N}$ & $\mathrm{Z}$ & $\log v_{\text {peak }}^{S}$ & SED class & AGN class \\
\hline FIRST J1301.7+4056 & $13: 01: 45.6$ & $+40: 56: 24$ & 1 & 0.649 & 13.04 & LSP* & BL Lac \\
\hline GB6 B1300+5804 & $13: 02: 52.4$ & $+57: 48: 37$ & 2 & 1.088 & 12.28 & LSP & bl.un \\
\hline RXS J1302.9+5056 & $13: 02: 55.5$ & $+50: 56: 17$ & 1 & 0.688 & 12.42 & LSP* & BL Lac \\
\hline MC2 $1307+12$ & 13:09:33.9 & $+11: 54: 24$ & 14 & 0.318 & 13.32 & LSP & BL Lac \\
\hline OP 313 & $13: 10: 28.6$ & $+32: 20: 43$ & 10 & 0.997 & 13.30 & LSP & bl.un \\
\hline PKS 1309-216 & $13: 12: 31.5$ & $-21: 56: 24$ & 1 & 1.491 & 15.46 & HSP & BL Lac \\
\hline RXS J1319.5+1405 & $13: 19: 31.7$ & $+14: 05: 34$ & 5 & 0.572 & 15.49 & HSP & BL Lac \\
\hline $\mathrm{J} 132247.40+3216$ & $13: 22: 47.3$ & $+32: 16: 08$ & 5 & 0 & 15.13 & HSP* & BL Lac \\
\hline RXS J1326.2+1230 & $13: 26: 17.6$ & $+12: 29: 58$ & 5 & 0.204 & 12.33 & LSP* & BL.gal.domin \\
\hline J132952.86+3154 & $13: 29: 52.8$ & $+31: 54: 11$ & 1 & 0 & 12.44 & LSP* & cand. \\
\hline SDSS J13338+5057 & $13: 33: 53.7$ & $+50: 57: 36$ & 1 & 1.362 & 14.32 & ISP & bl.un \\
\hline MS1332.6-2935 & $13: 35: 29.7$ & $-29: 50: 39$ & 1 & 0.513 & 14.86 & ISP & BL Lac \\
\hline RXS J1341.0+3959 & $13: 41: 05.0$ & $+39: 59: 45$ & 6 & 0.172 & 14.63 & ISP & BL.gal.domin \\
\hline J134916-141316 & $13: 49: 16.0$ & $-14: 13: 17$ & 1 & 0.253 & 13.20 & LSP* & BL Lac \\
\hline PKS1350+148 & $13: 53: 22.8$ & $+14: 35: 39$ & 2 & 0.807 & 13.57 & LSP & BL Lac \\
\hline RXS J1353.4+5601 & $13: 53: 28.0$ & $+56: 00: 56$ & 2 & 0.404 & 12.18 & LSP* & BL Lac \\
\hline MC $1400+162$ & $14: 02: 44.5$ & $+15: 59: 57$ & 12 & 0.244 & 14.44 & $\mathrm{ISP}^{*}$ & BL Lac \\
\hline MS 1402.3+0416 & $14: 04: 51.0$ & $+04: 02: 02$ & 5 & 0.344 & 15.83 & HSP & BL Lac \\
\hline MS 1407.9+5954 & $14: 09: 23.4$ & $+59: 39: 40$ & 1 & 0.496 & 15.04 & HSP* & BL Lac \\
\hline PKS 1407+022 & $14: 10: 04.6$ & $+02: 03: 06$ & 10 & 0 & 13.24 & LSP* & BL Lac \\
\hline RXS J1410.5+6100 & $14: 10: 31.7$ & $+61: 00: 10$ & 5 & 0.384 & 14.93 & ISP* & BL Lac \\
\hline PKS 1413+135 & $14: 15: 58.8$ & $+13: 20: 24$ & 11 & 0.247 & 12.96 & LSP & bl.un \\
\hline 2E $1415+2557$ & $14: 17: 56.6$ & $+25: 43: 25$ & 2 & 0.237 & 15.45 & HSP & BL Lac \\
\hline OQ 530 & $14: 19: 46.6$ & $+54: 23: 14$ & 4 & 0.153 & 13.98 & LSP & BL Lac \\
\hline SDSS J14202+0614 & $14: 20: 13.6$ & $+06: 14: 28$ & 1 & 0.625 & 13.49 & LSP* & BL Lac \\
\hline PKS 1424+240 & $14: 27: 00.5$ & $+23: 48: 00$ & 11 & 0.160 & 15.34 & HSP & BL Lac \\
\hline RXS J1436.9+5639 & $14: 36: 57.7$ & $+56: 39: 24$ & 1 & 0.150 & 16.88 & HSP & BL Lac \\
\hline PKS 1437-153 & $14: 39: 56.8$ & $-15: 31: 50$ & 7 & 0.636 & 13.46 & LSP & BL Lac \\
\hline 1ES $1440+122$ & $14: 42: 48.3$ & $+12: 00: 40$ & 6 & 0.162 & 16.35 & HSP & BL Lac \\
\hline RXS J14495+2746 & $14: 49: 32.6$ & $+27: 46: 21$ & 1 & 0.227 & 12.40 & LSP* & BL.gal.domin \\
\hline B2 $1451+26$ & $14: 53: 53.5$ & $+26: 48: 33$ & 3 & 0 & 13.12 & LSP* & bl.un \\
\hline RXS J1456.0+5048 & $14: 56: 03.7$ & $+50: 48: 25$ & 5 & 0.480 & 12.52 & LSP* & BL Lac \\
\hline RXS J1458.4+4832 & $14: 58: 27.0$ & $+48: 32: 46$ & 2 & 0.541 & 15.44 & HSP* & BL Lac \\
\hline B3 1456+375 & $14: 58: 44.8$ & $+37: 20: 22$ & 8 & 0.333 & 13.31 & LSP & BL Lac \\
\hline TXS $1459+480$ & $15: 00: 48.6$ & $+47: 51: 15$ & 1 & 1.059 & 13.15 & LSP & FSRQ \\
\hline PKS 1514+197 & $15: 16: 56.8$ & $+19: 32: 12$ & 19 & 0.650 & 12.97 & LSP & BL Lac \\
\hline PKS 1514-24 & $15: 17: 41.8$ & $-24: 22: 19$ & 4 & 0.048 & 14.19 & ISP & BL Lac \\
\hline PKS 1519-273 & $15: 22: 37.6$ & $-27: 30: 10$ & 5 & 1.294 & 12.79 & LSP & BL Lac \\
\hline 1ES $1533+535$ & $15: 35: 00.8$ & $+53: 20: 37$ & 5 & 0.890 & 16.99 & HSP & BL Lac \\
\hline MS $1534.2+0148$ & $15: 36: 46.8$ & $+01: 37: 59$ & 5 & 0.311 & 14.64 & ISP* & BL Lac \\
\hline 4C 14.60 & $15: 40: 49.4$ & $+14: 47: 45$ & 20 & 0.605 & 13.53 & LSP & BL Lac \\
\hline RXS J1542.9+6129 & $15: 42: 56.9$ & $+61: 29: 55$ & 2 & 0.117 & 14.64 & ISP & BL Lac \\
\hline RXS J1544.3+0458 & $15: 44: 18.7$ & $+04: 58: 22$ & 6 & 0.326 & 12.80 & LSP* & BL.gal.domin \\
\hline PG $1553+11$ & $15: 55: 43.1$ & $+11: 11: 24$ & 10 & 0.326 & 14.12 & ISP & BL Lac \\
\hline MYC $1557+566$ & $15: 58: 48.2$ & $+56: 25: 14$ & 1 & 0.300 & 14.21 & ISP & BL Lac \\
\hline CGRaBS J1603+1105 & $16: 03: 41.9$ & $+11: 05: 48$ & 7 & 0.143 & 13.46 & LSP & BL Lac \\
\hline PKS 1604+159 & 16:07:06.4 & $+15: 51: 34$ & 10 & 0.357 & 13.36 & LSP & BL Lac \\
\hline RXS J1610.0+6710 & $16: 10: 04.1$ & $+67: 10: 26$ & 1 & 0.067 & 16.20 & HSP* & BL Lac \\
\hline SDSS J16183+3632 & $16: 18: 23.5$ & $+36: 32: 01$ & 3 & 0.730 & 13.43 & LSP* & BL Lac \\
\hline NGC 6251 & $16: 32: 31.9$ & $+82: 32: 16$ & 3 & 0.025 & 13.73 & LSP & bl.un \\
\hline CGRaBS J1642-0621 & $16: 42: 02.1$ & $-06: 21: 23$ & 4 & 1.514 & 13.23 & LSP & BL Lac \\
\hline 1FGL J1647.4+4948 & $16: 47: 34.9$ & $+49: 50: 00$ & 1 & 0.047 & 13.70 & LSP & bl.un \\
\hline PKS 1648+015 & $16: 51: 03.6$ & $+01: 29: 23$ & 4 & 0.400 & 12.88 & LSP* & BL Lac \\
\hline MRK 501 & $16: 53: 52.2$ & $+39: 45: 36$ & 12 & 0.033 & 16.12 & HSP & BL Lac \\
\hline SDSS J16581+6150 & $16: 58: 08.3$ & $+61: 50: 02$ & 1 & 0.374 & 14.62 & ISP & BL Lac \\
\hline
\end{tabular}


Table 6 Blazar sample and some parameters. Continued.

\begin{tabular}{|c|c|c|c|c|c|c|c|}
\hline Source & R.A. (J2000) & Dec (J2000) & $\overline{\mathrm{N}}$ & $\mathrm{z}$ & $\log v_{\text {peak }}^{S}$ & SED class & AGN class \\
\hline PKS 1707-038 & $17: 10: 17.2$ & $-03: 55: 50$ & 9 & 1.920 & 12.78 & $\mathrm{LSP}^{*}$ & FSRQ \\
\hline PGC 59947 & $17: 15: 22.9$ & $+57: 24: 40$ & 1 & 0.027 & - & - & BL.gal.domin \\
\hline TEX 1714-336 & $17: 17: 36.0$ & $-33: 42: 08$ & 3 & 0 & 13.08 & LSP* & cand. \\
\hline PKS 1717+177 & $17: 19: 13.1$ & $+17: 45: 06$ & 11 & 0.137 & 13.33 & LSP* & BL Lac \\
\hline H $1722+119$ & $17: 25: 04.4$ & $+11: 52: 16$ & 6 & 0.018 & 16.01 & HSP & BL Lac \\
\hline BZB J1733+4519 & $17: 33: 28.8$ & $+45: 19: 50$ & 1 & 0.317 & - & - & BL.gal.domin \\
\hline OT 465 & $17: 39: 57.1$ & $+47: 37: 58$ & 1 & 0.950 & 13.19 & LSP & BL Lac \\
\hline NPM1G +19.0510 & $17: 43: 57.9$ & $+19: 35: 09$ & 7 & 0.083 & 15.76 & HSP & BL.gal.domin \\
\hline J1745-0753 & $17: 45: 27.1$ & $-07: 53: 03$ & 7 & 0 & 13.36 & LSP & BL Lac \\
\hline B2 $1743+39 C$ & $17: 45: 37.6$ & $+39: 51: 31$ & 3 & 0.267 & 15.15 & HSP & BL.gal.domin \\
\hline S4 1749+70 & $17: 48: 32.8$ & $+70: 05: 50$ & 3 & 0.770 & 13.78 & LSP & BL Lac \\
\hline PKS 1749+096 & $17: 51: 32.7$ & $+09: 39: 01$ & 17 & 0.322 & 12.41 & LSP & BL Lac \\
\hline RXS J1756.2+5522 & $17: 56: 15.9$ & $+55: 22: 18$ & 5 & 0.407 & 16.46 & HSP & BL Lac \\
\hline S5 $1803+78$ & $18: 00: 45.6$ & $+78: 28: 04$ & 5 & 0.680 & 13.55 & LSP & BL Lac \\
\hline 3 C 371 & 18:06:50.6 & $+69: 49: 28$ & 3 & 0.046 & 14.69 & ISP & BL Lac \\
\hline 4C 56.27 & $18: 24: 07.0$ & $+56: 51: 01$ & 3 & 0.664 & 13.19 & LSP & BL Lac \\
\hline 3C 380.0 & $18: 29: 31.7$ & $+48: 44: 46$ & 3 & 0.695 & 13.27 & LSP & bl.un \\
\hline 1H 1914-194 & $19: 17: 44.8$ & $-19: 21: 30$ & 1 & 0.137 & 15.41 & HSP & BL Lac \\
\hline S4 1926+61 & $19: 27: 30.4$ & $+61: 17: 31$ & 1 & 0 & 13.36 & LSP & BL Lac \\
\hline S5 2007+77 & $20: 05: 30.9$ & $+77: 52: 43$ & 6 & 0.342 & 13.53 & LSP & BL Lac \\
\hline $4 C+72.28$ & 20:09:52.3 & $+72: 29: 19$ & 2 & 0 & 12.90 & LSP & BL Lac \\
\hline PKS 2012-017 & $20: 15: 15.1$ & $-01: 37: 33$ & 6 & 0.520 & 14.43 & ISP & BL Lac \\
\hline S5 $2023+76$ & $20: 22: 35.5$ & $+76: 11: 26$ & 5 & 0.594 & 14.11 & ISP & BL Lac \\
\hline PKS 2029+121 & $20: 31: 54.9$ & $+12: 19: 41$ & 2 & 1.215 & 12.95 & LSP & bl.un \\
\hline PKS 2032+107 & $20: 35: 22.3$ & $+10: 56: 06$ & 6 & 0.601 & 14.03 & ISP & FSRQ \\
\hline 1ES 2037+521 & $20: 39: 23.5$ & $+52: 19: 49$ & 1 & 0.053 & 16.47 & HSP & cand. \\
\hline PKS 2047+039 & 20:50:06.2 & $+04: 07: 49$ & 12 & 0 & 13.08 & LSP & BL Lac \\
\hline S5 2051+74 & $20: 51: 33.7$ & $+74: 41: 40$ & 1 & 0 & 12.76 & LSP* & cand. \\
\hline GB6 J2116+3339 & $21: 16: 14.5$ & $+33: 39: 20$ & 1 & 0.350 & 15.33 & HSP & BL Lac \\
\hline PKS 2131-021 & $21: 34: 10.2$ & $-01: 53: 17$ & 14 & 0.557 & 13.19 & LSP & BL Lac \\
\hline MS 2143.4+0704 & $21: 45: 52.3$ & $+07: 19: 27$ & 5 & 0.237 & 14.58 & ISP & BL Lac \\
\hline PKS 2149+17 & $21: 52: 24.8$ & $+17: 34: 37$ & 15 & 0.871 & 13.38 & LSP & BL Lac \\
\hline PKS 2155-304 & $21: 58: 52.0$ & $-30: 13: 32$ & 3 & 0.116 & 15.97 & HSP & BL Lac \\
\hline BL LAC & $22: 02: 43.2$ & $+42: 16: 40$ & 19 & 0.069 & 13.39 & LSP & BL Lac \\
\hline B2 2214+24B & $22: 17: 00.8$ & $+24: 21: 46$ & 5 & 0.505 & 13.37 & LSP & BL Lac \\
\hline J221944+212056 & $22: 19: 44.1$ & $+21: 20: 53$ & 2 & 0.200 & 13.88 & LSP* & BL Lac \\
\hline PKS 2221-116 & $22: 24: 07.9$ & $-11: 26: 21$ & 2 & 0.115 & 13.41 & LSP & BL Lac \\
\hline PKS 2223-114 & $22: 25: 43.7$ & $-11: 13: 41$ & 5 & 0.997 & 13.04 & LSP* & BL Lac \\
\hline $3 C 446$ & $22: 25: 45.1$ & $-04: 56: 34$ & 14 & 1.404 & 13.32 & LSP & FSRQ \\
\hline FIRST J22279+0037 & $22: 27: 58.1$ & $+00: 37: 05$ & 1 & 0 & 14.41 & ISP & BL Lac \\
\hline RXS J2233.0+1335 & $22: 33: 01.1$ & $+13: 36: 02$ & 5 & 0.214 & 14.67 & ISP* & BL Lac \\
\hline PKS 2233-173 & $22: 36: 09.5$ & $-17: 06: 21$ & 1 & 0.647 & 13.57 & LSP & BL Lac \\
\hline PKS 2233-148 & $22: 36: 34.0$ & $-14: 33: 22$ & 2 & 0.325 & 13.08 & LSP & BL Lac \\
\hline B3 $2238+410$ & $22: 41: 07.2$ & $+41: 20: 11$ & 1 & 0.726 & 13.32 & LSP & BL Lac \\
\hline PKS 2240-260 & $22: 43: 26.4$ & $-25: 44: 30$ & 3 & 0.774 & 13.22 & LSP & BL Lac \\
\hline RGB J2243+203 & $22: 43: 54.7$ & $+20: 21: 03$ & 1 & 0 & 15.58 & HSP & BL Lac \\
\hline B3 $2247+381$ & $22: 50: 05.7$ & $+38: 24: 37$ & 1 & 0.119 & 15.93 & HSP* & BL Lac \\
\hline PKS 2251+006 & $22: 54: 04.4$ & $+00: 54: 20$ & 2 & 0 & 12.92 & LSP* & BL Lac \\
\hline PKS 2254+074 & $22: 57: 17.3$ & $+07: 43: 12$ & 7 & 0.190 & 13.76 & LSP* & BL Lac \\
\hline
\end{tabular}


Table 6 Blazar sample and some parameters. Continued.

\begin{tabular}{llllllll}
\hline Source & R.A. $(\mathrm{J} 2000)$ & Dec $(\mathrm{J} 2000)$ & $\mathrm{N}$ & $\mathrm{z}$ & $\log \gamma_{\text {peak }}^{S}$ & SED class & AGN class \\
\hline RXS J2304.6+3705 & $23: 04: 36.6$ & $+37: 05: 08$ & 7 & 0 & 15.88 & HSP & BL Lac \\
PGC 1465934 & $23: 13: 57.3$ & $+14: 44: 23$ & 1 & 0.163 & 15.42 & HSP & BL.gal.domin \\
Q J2319+161 & $23: 19: 43.4$ & $+16: 11: 50$ & 2 & 0 & 12.63 & LSP* & BL Lac \\
GB6 J2325+3957 & $23: 25: 17.8$ & $+39: 57: 37$ & 1 & 0 & 13.71 & LSP & BL Lac \\
1ES 2326+174 & $23: 29: 03.3$ & $+17: 43: 30$ & 5 & 0.213 & 15.62 & HSP* & BL Lac \\
Q J2338+212 & $23: 38: 56.4$ & $+21: 24: 41$ & 5 & 0.291 & 14.83 & ISP & cand. \\
MS 2336.5+0517 & $23: 39: 07.0$ & $+05: 34: 36$ & 2 & 0.740 & 15.95 & HSP* & BL Lac \\
B2 2337+26 & $23: 40: 29.0$ & $+26: 41: 56$ & 6 & 0.372 & 12.42 & LSP* & cand. \\
1FGL J2341.6+8015 & $23: 40: 53.7$ & $+80: 15: 13$ & 1 & 0.274 & 15.64 & HSP & BL Lac \\
MS 2342.7-1531 & $23: 45: 22.3$ & $-15: 15: 06$ & 1 & 0 & 16.18 & HSP* & FSRQ \\
MS J23492105+0534 & $23: 49: 21.0$ & $+05: 34: 40$ & 4 & 0.419 & 13.58 & LSP* & FSRQ \\
MS 2347.4+1924 & $23: 50: 01.7$ & $+19: 41: 52$ & 4 & 0.515 & 13.37 & LSP* & bl.un \\
RXS J2350.3-059 & $23: 50: 18.0$ & $-05: 59: 27$ & 1 & 0.515 & 13.89 & LSP* & cand. \\
PKS 2354-02 & $23: 57: 25.1$ & $-01: 52: 15$ & 7 & 0.812 & 13.38 & LSP & BL Lac \\
\hline
\end{tabular}

Col. 1 - source name, Col. 2-3 - R.A. and Dec (J2000.0), Col. 4 - number of observing epochs at RATAN, Col. 5 - redshift $z$ (BZCAT or Simbad),

Col. 6 - logarithm of the synchrotron peak frequency $v_{\text {peak }}^{S}$ in Hz, Col. 7 - SED class from BZCAT or Nieppola et al. (2006), we noted "*” blazars, for whic Col. 8 - AGN class from BZCAT 